\title{
Ondas em Água: Um Estudo da Altura e Ângulo de Quebra
}

\author{
Sebastião Romero Franco, \\ Departamento de Matemática, UNICENTRO, \\ Campus Irati-PR \\ 84500-000, Irati, PR \\ E-mail: romero@irati.unicentro.br, romero.unicentro@yahoo.com.br. \\ Leandro Farina \\ Departamento de Matemática Pura e Aplicada - UFRGS \\ 91509-900, Porto Alegre, RS e \\ Basque Center for Applied Mathematics - BCAM \\ 48009, Bilbao, País Basco, Espanha \\ E-mail: farina@mat.ufrg.br.
}

Resumo: Um método de aproximação de Fourier é utilizado para a modelagem e simulação de ondas completamente não lineares permanentes. O conjunto de equações não lineares resultante é resolvido pelo método de Newton. O empinamento de ondas é simulado, usando como base, comparações com dados experimentais. As alturas e ângulos de quebra são analisados até o limite de inadequação do método numérico. Os resultados se mostram bastante próximos dos critérios preditos pela teoria de ondas de superfície completamente não lineares e contribuem para proporcionar informações adicionais sobre o estudo da relação entre modelagem computacional e a teoria de ondas permanentes.

Palavras-chave: Ondas aquáticas não lineares, ondas permanentes, empinamento de ondas, método espectral.

\section{Introdução}

Ondas em água constitui um tópico que tem sido extensivamente estudados em diferentes domínios da ciência. Os fenômenos de empinamento e quebra de ondas possui um lugar de destaque neste contexto, visto que a energia de ondas está intrinsecamente associada à altura de onda. Métodos experimentais, analíticos e computacionais têm sido utilizados para a investigação destes fenômenos. Entre estes, os dados de campo apresentados por Hansen [9] e de medições em laboratório, originalmente obtidos por Eagleson [7] são dados clássicos para a validação de métodos numéricos.

Do ponto de vista analítico e computacional o artigo de Rienecker e Fenton [11] foi um dos primeiros a propor um método para a simulação de ondas aquáticas completamente não lineares permanentes. Denominado como método de Fourier, esta técnica não assume aproximações analíticas e a solução das equações não lineares governantes para a dinâmica de ondas é expressa por uma série de Fourier.

Abordagens com aproximações analíticas para o cálculo de ondas não lineares também têm sido empregadas. Freilich e Guza [5] usam variantes das equações de Boussinesq para estudar o empinamento de ondas. Fenton [4] deduziu expressões de quinta ordem baseadas na teoria de Stokes e apresentou resultados numéricos, comparando-os com dados experimentais. Neste mesmo contexto, Pihl et. al. [6] examinou o empinamento de ondas descritas por uma aproximação de sexta ordem da teoria de Stokes na presença de uma corrente. 
Para estudos da dinâmica de ondas não lineares com ênfase mais computacional, citamos o artigo de Drimer e Agnon [2] que utiliza o método de elemento de contorno e o trabalho de Ducrozet et. al. [3] que faz um estudo comparativo de dois métodos rápidos para o problema de ondas de superfície não lineares: o método espectral de ordem superior e método de diferenças finitas de alta ordem.

Neste artigo, resolvemos o problema de ondas de superfície completamente não lineares permanentes pelo método de Fourier combinado com o método de Newton. Nenhuma aproximação analítica é feita e assumimos que em um fundo com declividade, as ondas em qualquer profundidade se comportam como se o fundo fora horizontal. A aproximação por séries de Fourier permitiu o cálculo de soluções acuradas, mesmo para ondas altas e para todos comprimentos de onda, exceto para o limite de um sóliton. Exploramos estas características para estudar com certo nível de detalhe o fenômeno de empinamento de ondas.

\section{Modelo Matemático}

A descrição matemática da propagação de ondas de gravidade na superfície da água usualmente requer a consideração de algumas hipóteses das propriedades da água e do movimento realizado por ela. Para isso, considera-se um fluido homogêneo, incompressível e com movimento irrotacional onde a principal força restauradora é devido à gravidade. Além disso, a viscosidade e a tensão superficial são desprezíveis. Ademais, não consideraremos a forçante do vento.

Para o problema de ondas bidimensional permanentes, a formulação do problema é desenvolvida em termos da função corrente $\psi$. Para isso, assume-se que a coordenada $x$ indica a direção horizontal, $y$ indica a direção vertical e a origem do sistema cartesiano encontra-se no fundo da água. Denotamos por $(u, v)$ as componentes do vetor velocidade $\mathbf{u}$ e a função corrente $\psi$ é definida de tal forma que $u=\frac{\partial \psi}{\partial y}$ e $v=-\frac{\partial \psi}{\partial x} . \psi(x, y)$ satisfaz a equação da continuidade, ou seja, $\nabla \cdot \mathbf{u}=0$ e a equação de Laplace

$$
\frac{\partial^{2} \psi}{\partial x^{2}}+\frac{\partial^{2} \psi}{\partial y^{2}}=0 \quad \text { em } 0<y<\eta(x) .
$$

As condições de contorno que devem ser satisfeitas pela função de corrente são

$$
\begin{array}{r}
\psi(x, 0)=0, \\
\psi(x, \eta(x))=-Q,
\end{array}
$$

na origem (fundo) e na superfície livre $y=\eta(x)$, respectivamente.

Na equação (3), $Q$ é a vazão volumétrica por unidade de comprimento de crista na direção normal ao plano $x y$. Neste caso, assume-se que o fluxo se movendo da direita para a esquerda estará no sentido negativo. Na superfície livre, a pressão é constante, assim, pela equação de Bernoulli, tem-se:

$$
\frac{1}{2}\left[\left(\frac{\partial \psi}{\partial x}\right)^{2}+\left(\frac{\partial \psi}{\partial y}\right)^{2}\right]+\eta=R,
$$

onde $R$ é a energia total do sistema.

\section{Aproximação de Ondas Completamente Não Lineares Perma- nentes}

A aproximação da solução será obtida através de um método espectral, como visto em Dommermuth e Yue [1], combinado com o método de Newton. Para isso, as variáveis foram adimensionalizadas com respeito à aceleração da gravidade, $g$ e à profundidade média da água, $\bar{\eta}$. 
Para resolver o problema numericamente, considerando a simetria da onda pela crista, $\psi(x, y)$ pode ser discretizada ao longo de $N+1$ pontos, da crista ao cavado da onda, e expandida como [8]

$$
\begin{aligned}
B_{0} \eta_{m}+\sum_{j=1}^{N} B_{j} \frac{\operatorname{senh} j k \eta_{m}}{\cosh j k D} \cos \left(\frac{j m \pi}{N}\right)+Q & =0 \\
\frac{1}{2} u_{m}^{2}+\frac{1}{2} v_{m}^{2}+\eta_{m}-R & =0
\end{aligned}
$$

para $m=0,1, \ldots, N$, onde

$$
\begin{aligned}
& u_{m}=B_{0}+k \sum_{j=1}^{N} j B_{j} \frac{\cosh j k \eta_{m}}{\cosh j k D} \cos \left(\frac{j m \pi}{N}\right), \\
& v_{m}=k \sum_{j=1}^{N} j B_{j} \frac{\operatorname{senh} j k \eta_{m}}{\cosh j k D} \operatorname{sen}\left(\frac{j m \pi}{N}\right),
\end{aligned}
$$

Onde $D$ é um nível de referência arbitrária.

Como a altura média admensionalizada da onda é unitária, podemos escrever

$$
\frac{1}{2 N}\left[\eta_{0}+\eta_{N}+2 \sum_{j=1}^{N-1} \eta_{j}\right]-1=0 .
$$

A altura $H$ é a diferença entre a elevação da crista $\eta_{0}$ e do cavado $\eta_{N}$ da onda, assim,

$$
\eta_{0}-\eta_{N}-H=0
$$

A equação que envolve o período $\tau$ da onda, por sua vez, é dada por,

$$
k c \tau-2 \pi=0,
$$

onde $c$ é a velocidade de fase da onda, $k$ é o número de onda e $\lambda$ é o comprimento de onda.

Em um regime permanentes de ondas, a vazão volumétrica $Q$ é igual a velocidade média com que a partícula de fluido se move. Portanto, a velocidade de transporte de massa, dado por $c_{s}$ pode ser expressa por

$$
c-c_{s}-Q=0 \text {. }
$$

Por fim, as $2 N+6$ equações (5), (6),(7)-(10) formam um sistema fechado para as variáveis $\left(\eta_{j}, B_{j}(j=0,1, \ldots, N), k, Q, R, c\right)$.

\section{Resultados}

Para a comparação com os experimentos, usaremos dados de ondas obtidos em praias e em tanques de teste. Estes experimentos foram originalmente relatados nos artigos de Hansen e Svendsen [9] e posteriormente utilizados como comparação com outros métodos e teorias. Veja, por exemplo [11] e [13].

Os dados experimentais foram obtidos a partir de declividades de praia uniformes de 1/35 [9]. Os dados foram coletados até a quebra da onda, ponto este em que a modelagem presente neste trabalho não é mais aplicável.

Para a realização das simulações usamos os parâmetros $D=1$, o número de termos $N$ para as expansões de Fourier em (5) e (6) igual a 16 e a taxa de declividade do fundo $r=0,999$. Para efeito de comparação, a linha sólida indica os dados obtidos a partir das simulações numéricas com o presente método e os pontos indicam os dados obtidos experimentalmente por [9].

Nas figuras 1, mostramos a altura de onda em função da profundidade. Neste caso foram simulado três ondas, (a), (b) e (c), respectivamente. Para a realização das simulações foram 

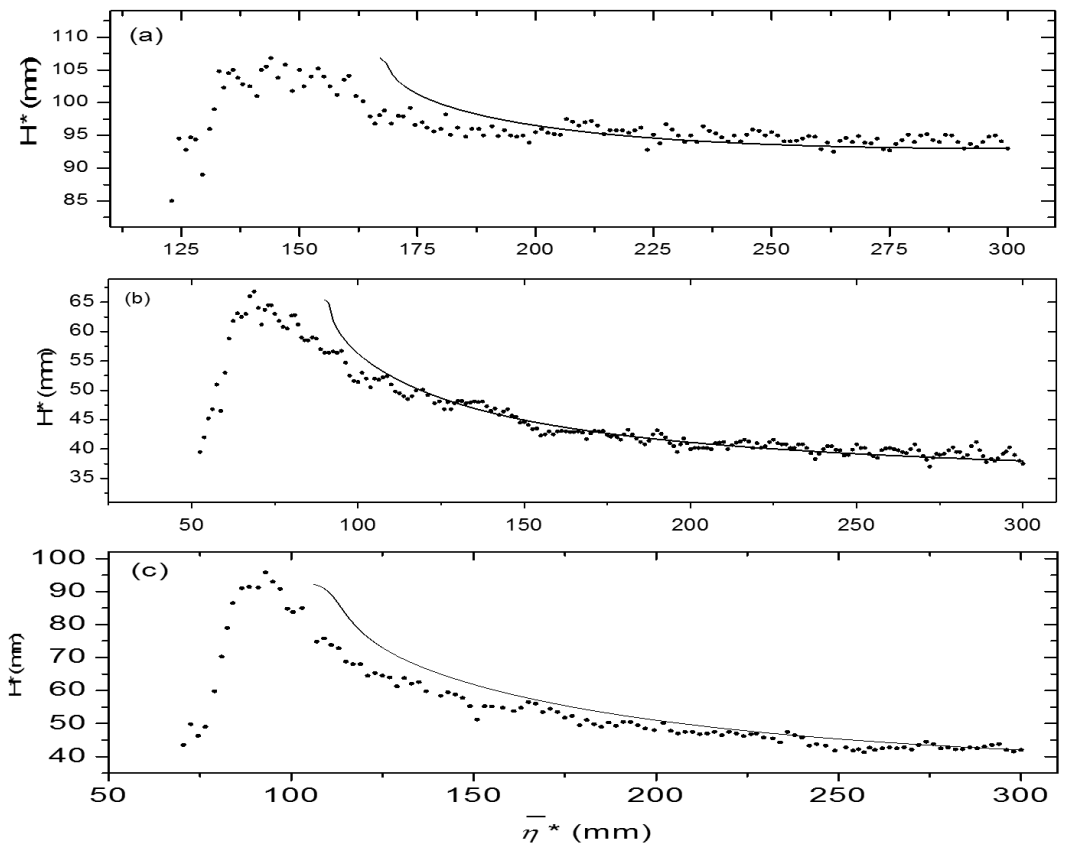

Figura 1: Altura da onda em função da profundidade da água.

fornecido os valores da altura inicial e período inicial da onda. No caso da onda (a), a altura inicial foi $H_{0}=93 \mathrm{~mm}$ e o período inicial $\tau_{0}=1 \mathrm{~s}$, enquanto que nas ondas (b) e (c), os valores foram $H_{0}=39 \mathrm{~mm}$ e $\tau_{0}=1,67 \mathrm{~s}$ para a onda (b) e $H_{0}=12 \mathrm{~mm}$ e $\tau_{0}=3,33 \mathrm{~s}$ para a onda (c).

A onda (b) é mais baixa e possui um período inicial maior que a onda (a), enquanto que a onda (c) apresenta um período significativamente maior que as anteriores. Com isso, observamos graficamente que as simulações apresentam uma boa concordância com os dados experimentais. Em particular, o empinamento das ondas é notável, com a diminuição da profundidade, em todos os casos até valores bem próximos ao ponto de quebra da onda.

\subsection{Altura e Ângulo de Quebra}

Ondas propagando na zona de empinamento de onda, em águas intermediárias, tornam-se instáveis e quebram quando a velocidade da partícula de água na crista da onda torna-se igual ou superior à velocidade de fase da onda. Na quebra, a altura da onda é limitada pela profundidade e comprimento de onda. Para uma dada profundidade e período de onda, há um limite máximo para a altura da onda, chamado de altura de quebra da onda. De acordo com a teoria de Stokes, em águas intermediárias, a altura de quebra é $\frac{H}{\bar{\eta}}=0,78$ [12, p. 06].

Em nosso modelo, $\eta(x)$ é por definição unicamente definida para cada $x$. Portanto, obrigatoriamente o método utilizado para solução não se aplicará até o limite físico da quebra da onda. Antes da quebra, a superfície da onda se torna multivaluada e portanto não modelável por uma função. No caso específico do método de Newton, este divergirá.

Definimos como altura de quebra de onda computacional e denotamos por $\left(\frac{H^{*}}{\bar{\eta}^{*}}\right)_{b}$, a última altura para a qual houve convergência do método de Newton que resolve o modelo computacional.

A figura 2 mostra a evolução parâmetro altura por profundidade, dado por $H^{*} / \bar{\eta}^{*}$ e a altura de quebra de onda computacional $\left(\frac{H^{*}}{\bar{\eta}^{*}}\right)_{b}$ em função do parâmetro $\bar{\eta} / \lambda$, a profundidade relativa ao comprimento de onda. Nesta figura estão representados os casos referentes às ondas (a), (b) e (c) descritas na figuras 1 .

As curvas 2(a) e 2(b) quebraram a uma profundidade relativa de $\frac{\bar{\eta}}{\lambda}=0,13256$ e $\frac{\bar{\eta}}{\lambda}=0,0516$, respectivamente. Isso indica que o processo de empinamento até a quebra da onda ocorreu em águas intermediárias. A onda representada na figura 2(c) é significativamente mais longa 


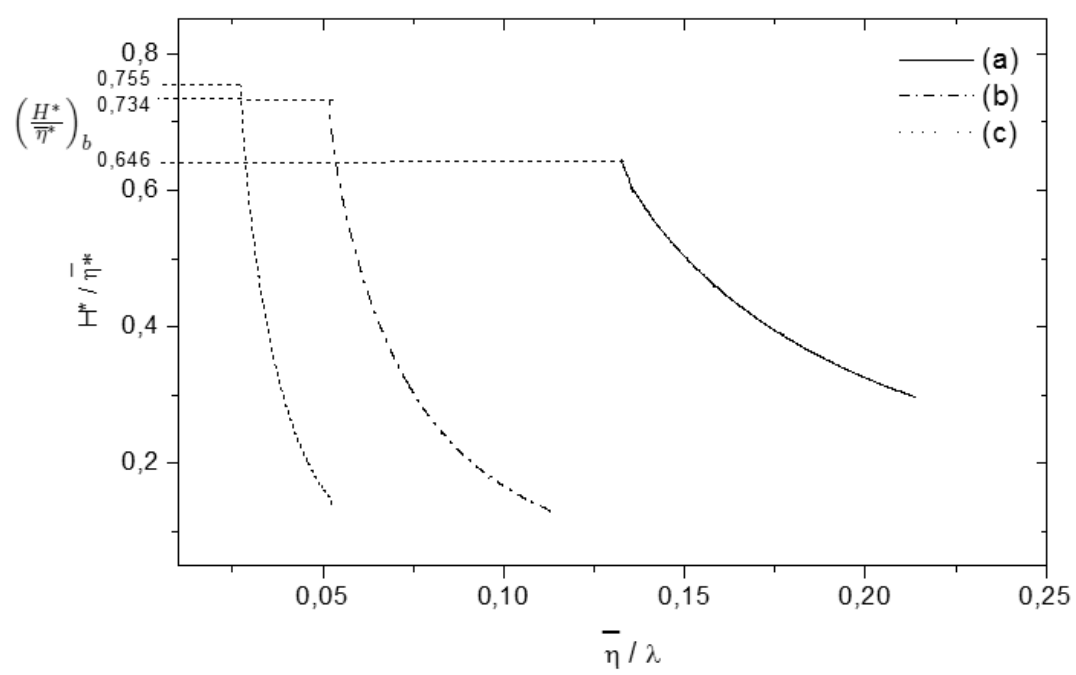

Figura 2: A evolução do parâmetro $H^{*} / \bar{\eta}^{*}$ e a altura de quebra de onda computacional.

e apresenta praticamente todo o seu processo de empinamento em águas rasas, quebrando em uma profundidade relativa de $\frac{\bar{\eta}}{\lambda} \approx 0,027$. Observamos nas figuras que que o modelo e método computacional preveem uma altura de quebra muito próxima da observada experimentalmente.

Usando a teoria de Stokes, pode-se mostrar [10] que o ângulo de quebra de uma onda é $120^{\circ}$. Usaremos o método espectral descrito neste trabalho para estimar o ângulo de quebra computacional $\alpha$, ou seja, aquele até quando podemos obter convergência do método de Newton usado para resolver o sistema de equações não lineares que governam as ondas em água.

A figura 3 mostra o perfil e o ângulo de quebra da onda (b) descrita na figura 1. A figura é dupla: a parte (I) mostra o perfil da onda no instante inicial, com $\bar{\eta}_{0}^{*}=300 \mathrm{~mm}, H_{0}^{*}=39 \mathrm{~mm} \mathrm{e}$ $\tau_{0}^{*}=1,67$ s e a parte (II), no momento da quebra de onda computacional com uma profundidade de $\bar{\eta}^{*}=93,52 \mathrm{~mm}$. Uma linha reta horizontal é incluída em todas as figuras para representar a profundidade média.

Pode-se verificar que o modo com que a onda empina-se depende diretamente do comprimento de onda. Para ondas de maior comprimento, temos que o cavado da onda torna-se mais longo horizontalmente. Com isto, a crista tem uma elevação mais acentuada no comprimento de onda.

Os ângulos de quebra computacionais das ondas (a), (b) e (c) descritas nas figuras 1 foram $134,12^{\circ}, 142,64^{\circ}$ e $126,20^{\circ}$, respectivamente.
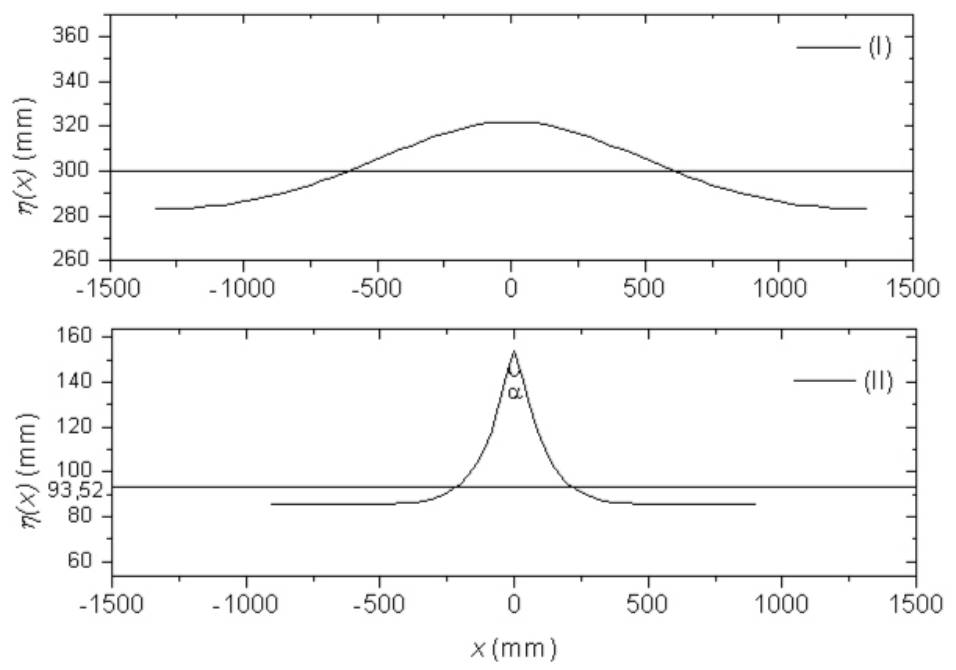

Figura 3: Perfil das ondas no instante inicial e no momento da quebra 


\section{Conclusões}

Um método de aproximação de Fourier foi utilizado para a modelagem e simulação de ondas completamente não lineares permanentes. O conjunto de equações não lineares resultante foi resolvido pelo método de Newton. Após uma cuidadosa readimensionalização, supomos que em um fundo inclinado, as ondas, em qualquer profundidade, se comportam como em fundos horizontais, um método iterativo foi descrito para o estudo do empinamento de ondas.

Um conjunto de dados experimentais foi utilizado para definir os estados iniciais de 3 casos de estudo. A partir destes, pode-se validar o método que apresentou excelente acordo com as medições. Uma análise dos chamados altura de quebra de onda e ângulo de quebra computacionais pode ser feita e valores foram obtidos para efeito de comparação entre as simulações e os critérios teóricos de altura e ângulo de quebra. Estes resultados, portanto contribuem para o conhecimento das associações existentes entre métodos de aproximação analítico-computacionais e a teoria de ondas de superfície não lineares.

\section{Referências}

[1] D. G. Dommermuth, D. K. P. Yue, A high-order spectral method for the study of nonlinear gravity waves, Journal of Fluid Mechanics 184, (1987), 267-288.

[2] N. Drimer, Y. Agnon, An improved low-order bounday element method for breaking surface waves, Journal of Wave Motion 43, (2006), 241-258.

[3] G. Ducrozet, H. B. Bingham, A. P. Engsig-Karup, F. Bonnefoy, P. Ferrant, A comparative study of two fast nonlinear free-surface water waves models, Int. J. Num. Methods in Fluids 69, (2012), 1818-1834.

[4] J. F. Fenton, A fifth-order Stokes theory for stead waves, J. Waterway, Port, Coastal, Ocean Engng. 111, (1985), 216-234.

[5] M. H. Freilich, R. T. Guza, Nonlinear Effects on Shoaling Surface Gravity Waves, Phil. Trans. R. Soc. Lond. A 311, (1984), 1-41.

[6] J. H. Pihl, H. Bredmose and J. Larsen, Shoaling of sixth-order waves on a current, Ocean Engng. 28 , (2001), 667-687.

[7] P. S. Eagleson, Properties of shoaling waves by theory and experiment, Transactions, American Geophysical Union 37, (1956), 565-572.

[8] J. D. Fenton, The Numerical Solution of Stedy Water Wave Problems, Computers and Geosciences 14, No. 3 (1988), 357-368.

[9] J. B. Hansen, I. A. Svendsen, Regular wave in shoaling water: experimental data, em "Inst. of Hydrodynamics and Hydraulic Engng. Tech. Univ. of Denmark",series paper No. 21 , 1979 .

[10] B. Kinsman, "Wind Waves - their generation and propagation on the ocean surface", Prentice-Hall, Inc.: New Jersey, 1965.

[11] M. M. Rienecker, J. D. Fenton, A Fourier Approximation Method for Steady Water Waves, Journal of Fluid Mechanics 104, (1981), 119-137.

[12] Secretariat of the World Meteorological Organization, "Guide to Wave Analysis and Forecasting", No. 702, Geneva - Switzerland, 1998.

[13] M. Stiassnie, D. H. Peregrine, Shoaling of finite-amplitude surface waves on water of slowlyvarying depth, Journal of Fluid Mechanics 97, (1980), 783-805. 